\title{
ÍNDICE DE ACIDEZ DEL ACEITE DE PEZ EN LA NUTRICIÓN DE ALEVINES DE PACÚ PIARACTUS MESOPOTAMICUS
}

\author{
ACIDITY FISH OIL INDEX IN THE NUTRITION OF PACU \\ PIARACTUS MESOPOTAMICUS JUVENILES
}

\author{
Lui, T.A. ${ }^{*}$, Bittencourt, F. ${ }^{2}$, Neu, D.H. ${ }^{1 \mathrm{~A}}$, Dallagnol, J.M. ${ }^{1 \mathrm{~B}}$, Boscolo, W.R. ${ }^{1 \mathrm{C}}$ y Feiden, A. ${ }^{1 \mathrm{D}}$ \\ 1Universidad Estadual del oeste del Paraná. Toledo, PR. Brasil. *taty_lui@hotmail.com; \\ Adacley_pesca@hotmail.com; Bjack.y.line@hotmail.com; ${ }^{\mathrm{c}}$ wilsonboscolo@hotmail.com; \\ Daldifeiden@gmail.com \\ ${ }^{2}$ CAUNESP. Jaboticabal, SP. Brasil. bitanca@hotmail.com
}

\section{Palabras clave adicionales}

Alimentación. Especies nativas. Piscicultura.

\section{RESUMEN}

El objetivo de este trabajo fue evaluar la influencia de los distintos índices de acidez del aceite de pescado en la nutrición de alevines de pacú Piaractus mesopotamicus. Para esto, 200 alevines con un peso y longitud total iniciales de $10,53 \pm 0,65 \mathrm{~g}$ y $8,74 \pm 0,13 \mathrm{~cm}$, respectivamente, fueron distribuidos en un diseño completamente al azar en 20 tanques de malla de $0,15 \mathrm{~m}^{3}$ de volumen útil situados dentro de un tanque de $25 \mathrm{~m}^{3}$. Los tratamientos consistieron en una dieta comercial extrusionada bañada con aceite de soja (control) con un índice de acidez de 0,24 y aceite de pescado con índices de 1,$48 ; 6,40$ y 9,85. Los peces fueron alimentados cuatro veces al día a saciedad aparente. Al final del periodo de estudio se analizaron el desempeño productivo, la composición química y la bioquímica sanguínea de los animales. Ninguno de los parámetros analizados sufrió cambios debidos a la acidez del aceite utilizado, por lo que el aceite de pescado puede ser suministrado a los animales de esta especie independientemente de su $\mathrm{pH}$.

\section{SUMMARY}

The aim of this study was to evaluate the effects of fish oil acidity on the growth of pacu (Piaractus mesopotamicus) juveniles. Two hundred juveniles with $10.53 \pm 0.65 \mathrm{~g}$ of weight and $8.74 \pm 0.13 \mathrm{~cm}$ of total length, were distributed in a totally randomized design in $20,0.15 \mathrm{~m}^{3}$ mesh-

\author{
Additional KEYWORDS \\ Feeding. Fish farming. Native species.
}

made tanks placed in a $25 \mathrm{~m}^{3}$ holding tank. Extruded commercial diets sprayed with soybean oil (control) with acidity index of 0.24 and fish oil with acidity index of $1.48,6.40$ and 9.85 were used. The fish were fed four times a day until apparent satiety. Productive performance, centesimal composition and blood biochemistry were analysed. At the end of the feeding period none of the analyzed parameters was affected by the use of acidified fish oil. Therefore, fish oil may be supplied to the pacu juveniles regardless of their $\mathrm{pH}$.

\section{INTRODUCCIÓN}

La acuicultura ha crecido de forma constante en Brasil y según datos del Ministerio de Pesca y Acuicultura (MPA, 2010) hubo un incremento superior al $15 \%$ entre los años de 2007 y 2009, siendo superior a cualquier otra actividad agropecuaria.

El principal motivo de este desarrollo está relacionado con la concienciación del consumo de pescado, influenciado también por las fuertes políticas del sector. Sin embargo, algunos obstáculos todavía están relacionados con la producción intensiva de peces, y uno de ellos es la alimentación, que es responsable de más del $50 \%$ del coste total (Cheng et al., 2003).

La dieta juega un papel fundamental 
durante el engorde, porque cuando el pez es cultivado en tanques de malla o hapas, permanece confinado sin que pueda alimentarse de organismos naturales, siendo necesario suministrar dietas balanceadas en cuanto a los niveles de energía, proteína, grasa, carbohidratos y contenido de aminoácidos para que no se produzcan mortalidades o se reduzca el crecimiento.

La energía de la dieta es debida principalmente al contenido en lípidos, proteínas y carbohidratos, y es esencial para el crecimiento, mantenimiento y reproducción de los peces (Graeff y Tomazelli, 2007). Como fuente de energía se incluyen en los piensos sobre todo aceites vegetales o de pescado ya que son incorporados en los peces con un alto grado de eficiencia, digestibilidad, y disponibilidad, además de ser compuestos ricos en ácidos grasos, incluyendo los de la serie $\omega-3$.

Los aceites de soja y pescado son los más utilizados en los piensos para organismos acuáticos. Sin embargo algunas características de estos productos pueden variar en función del tiempo de conservación o de la calidad inicial, una de esas características es la acidez, que además de modificar el precio del producto, también repercute en el aprovechamiento por parte de la industria (Araújo et al., 2006) y por parte del animal. Para que un aceite sea clasificado como aceite industrial, la materia prima necesita tener un índice de acidez inferior a 3, valores más grandes pueden comprometer la calidad del producto.

En este sentido, la búsqueda de ingredientes eficaces para el engorde de peces cultivados ha sido estudiada por un gran número de investigadores para distintas especies de peces, principalmente aquellos que presentan un gran potencial para el cultivo como es el caso del pacú ( $P$. mesopotamicus).

El pacú, originario de la cuenca de los ríos Paraná, Paraguay y Uruguay, es un representante del superorden Ostariophysi, donde se incluyen los peces de mayor valor comercial (Urbinati y Gonçalves, 2005), presenta una buena adaptación para el cultivo en tanques de malla, y un bajo requerimiento de proteína en su dieta (Signor et al., 2010).

Por ser un pez con gran versatilidad para la pesca y la acuicultura, así como por la disponibilidad de diferentes fuentes de aceites, el objetivo de este estudio fue determinar la influencia de los índices de acidez de los aceites en el desempeño zootécnico, en la composición química y la bioquímica sanguínea de los peces alimentados con raciones que contienen estos ingredientes.

\section{MATERIAL Y MÉTODOS}

El experimento se llevó a cabo en el invernadero experimental del grupo de estudios del manejo en la acuicultura/GEMAq de la Universidad Estadual del oeste del Paraná-UNIOESTE/Campus de Toledo (Brasil). Doscientos pacúes juveniles con peso y longitud total inicial media de 10,53 $\pm 0,65$ g y $8,74 \pm 0,13 \mathrm{~cm}$, respectivamente, se distribuyeron en 20 tanques de malla de plástico de $1 \times 4 \mathrm{~mm}$ con $0,15 \mathrm{~m}^{3}$ de volumen útil (40x40x70 cm de largo, ancho y altura/profundidad, respectivamente), dispuestos en un tanque de concreto con $25 \mathrm{~m}^{3}$ de capacidad con aireación constante. La distribución de los tratamientos fue en un diseño completamente aleatorio con cuatro tratamientos y cinco repeticiones.

Se utilizó un pienso comercial extrusionado con un $32 \%$ de proteína bruta (tabla I), sometido a un baño de aceite $(6 \%)$ con diferentes índices de acidez (IA). Los aceites utilizados para el baño fueron aceite de soja (IAS 0,24), y tres aceites de tilapia con índices de acidez distintos (IA1: 1,48), (IA2: 6,40), (IA3: 9,85).

Los distintos piensos así formulados fueron pesados y almacenados en recipientes de plástico. Las cantidades sobrantes de cada recipiente se pesaron al final del estudio permitiendo así evaluar el consumo total durante la realización del experimento. 


\section{ACIDEZ DEL ACEITE DE PEZ EN LA ALIMENTACIÓN DE PACÚ}

Tabla I. Análisis proximal de la dieta comercial extrusionada enriquecida con aceites que contienen diferentes índices de acidez y usada para la alimentación de alevines de pacú (Piaractus mesopotamicus). (Proximal analysis of the commercial extruded diet supplemented with oils containing different levels of acidity and utilized to feed pacu (Piaractus mesopotamicus) juveniles).

\begin{tabular}{lr} 
Nutrientes & (\%) \\
\hline Humedad (máx.) & 12,0 \\
Proteína bruta (mín.) & 32,0 \\
Extracto etéreo (mín.) & 7,0 \\
Fibra (máx.) & 9,0 \\
Cenizas (máx.) & 12,0 \\
Calcio (máx.) & 2,0 \\
Fósforo (máx.) & 1,0 \\
Premix de minerales y vitaminas $^{1}$ & 1,0 \\
\hline
\end{tabular}

'Suplemento de minerales y vitaminas: $\mathrm{Mn} 30 \mathrm{mg}$; Zn 160 mg; Fe 80 mg; Cu 20 mg; I 5 mg; Se 0,4 mg; Vit A 20000 Ul; Vit D3 6400 UI; Vit E 160 mg; Vit K3 $20 \mathrm{mg}$; Vit B1 $10 \mathrm{mg}$; Vit B2 $15 \mathrm{mg}$; Vit B6 $20 \mathrm{mg}$; Vit B12 $200 \mu \mathrm{g} ;$ Vit C 500 mg; Ác. Fólico 1,5 mg; Ác. Pantoténico $120 \mathrm{mg}$; Niacina 300 mg; Inositol 250 mg; Colina 1800 mg; Lisina 4\%; Biotina 1,4 mg/kg del producto.

Los animales fueron alimentados cuatro veces al día (08 h 00 min; 11 h 00 min; 14 h $00 \mathrm{~min}$; 17 h $00 \mathrm{~min}$ ) hasta saciedad aparente durante 40 días

Los parámetros físico-químicos del agua como pH, oxígeno disuelto (mg. $\left.\mathrm{L}^{-1}\right)$ y conductividad eléctrica $\left(\mu \mathrm{S} . \mathrm{cm}^{-1}\right)$ fueron monitoreados semanalmente usando potenciómetros digitales portátiles y la temperatura del agua $\left({ }^{\circ} \mathrm{C}\right)$ medida dos veces al día, por la mañana y por la tarde, con un termómetro.

Al final del período experimental los animales se mantuvieron en ayuno durante 24 horas para el vaciamiento del tubo digestivo. Tres peces de cada unidad experimental se utilizaron para la colecta de sangre. Previamente los animales fueron anestesiados con Eugenol® (solución de aceite de clavo) a $75 \mathrm{ml} . \mathrm{L}^{-1}$ y transportados hasta el Laboratorio de Tecnología Pesquera del GEMAq. Las muestras de sangre se tomaron con ayuda de una jeringa desechable, centrifugada durante 10 minutos a 800 RPM para separar el suero que fue utilizado para realizar los análisis de colesterol $\left(\mathrm{mg} \mathrm{dl}^{-1}\right)$ y triglicéridos $\left(\mathrm{mg} \mathrm{dl}^{-1}\right)$ mediante colorimetría utilizando el Kit Gold Analise $®$ (Belo Horizonte/MG, Brasil).

Posteriormente se procedió al recuento de todos los animales y a la estimación del peso y longitud individual. Los parámetros evaluados fueron peso final (PF), longitud total final (LTF), supervivencia (SOB), ganancia de peso (GP), conversión alimenticia (CA), tasa de crecimiento específico (TCE), grasa perivisceral $(\mathrm{GV})$ e índice hepatosomático (IHS). Para esto, los animales fueron pesados en balanza digital (precisión de 0,0001), abiertos en la parte ventral y eviscerados. Las vísceras se colocaron en placas de Petri donde se realizó la separación de la grasa y del hígado y este material fue pesado en la balanza analítica.

Las muestras fueron separadas y almacenadas bajo refrigeración $\left(-20^{\circ} \mathrm{C}\right)$ para la realización de los análisis de composición química mediante los métodos descritos en AOAC (2005) donde fueron evaluados la humedad (UM), materia seca(MS), proteína bruta (PB), cenizas (CZ) y lípidos (LP).

Los resultados de los análisis fueron tabulados y sometidos a pruebas de normalidad y al análisis de la varianza utilizando el programa estadístico Statistic 7.1.

\section{RESULTADOSYDISCUSIÓN}

Los valores de los parámetros de temperatura, conductividad, contenido de oxígeno y $\mathrm{pH}$ del agua durante el período experimental fueron $19,54 \pm 1,36^{\circ} \mathrm{C} ; 146,66 \pm 3,66$ $\mu \mathrm{S} . \mathrm{cm}^{-1} ; 2,45 \pm 0,05 \mathrm{mg} . \mathrm{L}^{-1}$ y $7,00 \pm 0,16$ respectivamente. Estos valores están de acuerdo con los recomendados para la producción de peces tropicales (Boyd, 1990; Sipaúba-Tavares, 1995). 


\section{LUI, BITTENCOURT, NEU, DALLAGNOL, BOSCOLO Y FEIDEN}

Los datos del desempeño como PI, PF, LTF, SOB, GP, CA, TCE, GV y IHS, no presentaron diferencias estadísticas en relación a los diferentes índices de acidez del aceite utilizado en el pienso (tabla II).

Entre todas las fuentes de aceite utilizadas, ninguna presentó efectos negativos sobre la supervivencia o crecimiento de los pacúes. Los peces tienen una alta capacidad para utilizar diferentes tipos y fuentes de aceites y grasas, sin embargo, cuando estos alimentos son procesados inadecuadamente, o suministrados en exceso puede haber una acumulación indeseable de grasa perivisceral, que reduce el rendimiento del filete y en consecuencia el valor comercial del pescado (Meurer et al., 2002). En el presente estudio, la ración proporcionada a los peces correspondió muy probablemente a satisfacer las necesidades de manutención de los peces sin que se produjera la acumulación de grasa perivisceral, con índices que varían desde 1,14 hasta 1,29\% del peso corporal. Bittencourt et al. (2010) observaron cantidades de grasa visceral alrededor de $7 \%$ del peso de pacúes adultos.
Boscolo et al. (2004) evaluaron el efecto de la inclusión de aceite de soja en el engorde de tilapia sin observar diferencias en el desempeño de los animales cuando el aceite era incorporado entre el 1,90 y el 5,90\% en la dieta, cantidades similares a las usadas en el presente trabajo. Aunque el aprovechamiento de estas fuentes de energía es diferente en función de la especie, las fases de desarrollo en que se encuentran y los hábitos alimentarios, el pacú utilizó con eficacia incluso el $6 \%$ de aceite incluido en la dieta ya formulada, no observándose pérdidas en el desempeño de los animales.

El efecto del índice de acidez de los aceites en la nutrición de los peces ha sido poco investigado $\mathrm{y}$, por consiguiente, el conocimiento sobre los trastornos que pueden surgir en los animales es incipiente. Existe una gran información sobre los efectos de diferentes fuentes de aceite, sea vegetal, animal o su mezcla, en distintas especies de peces, sin embargo, el índice de acidez del aceite no se ha tenido en cuenta, lo que puede estar relacionado con las buenas fuentes de ingredientes utilizadas por la

Tabla II. Parámetros del desempeño productivo del pacú (Piaractus mesopotamicus), alimentados con raciones que contienen aceite de diferentes niveles de acidez. (Productive performance parameters of Piaractus mesopotamicus fed with diets containing oils with different levels of acidity).

\begin{tabular}{lcccc}
\hline & \multicolumn{4}{c}{ Índices de acidez del aceite } \\
& 0,24 & 1,48 & 6,40 & 9,85 \\
\hline PI $(\mathrm{g})$ & $10,41 \pm 0,71$ & $10,78 \pm 0,72$ & $10,78 \pm 0,45$ & $10,15 \pm 0,65$ \\
PF $(\mathrm{g})$ & $26,06 \pm 2,35$ & $27,80 \pm 2,81$ & $28,52 \pm 1,27$ & $27,64 \pm 2,08$ \\
LTF $(\mathrm{cm})$ & $10,28 \pm 0,33$ & $10,58 \pm 0,32$ & $10,54 \pm 0,24$ & $10,68 \pm 0,28$ \\
SOB $(\%)$ & 100 & 100 & 100 & 100 \\
GP $(\mathrm{g})$ & $15,65 \pm 2,80$ & $17,03 \pm 3,21$ & $17,74 \pm 0,95$ & $17,49 \pm 1,76$ \\
CA & $1,15 \pm 0,12$ & $1,13 \pm 0,20$ & $1,03 \pm 0,02$ & $1,07 \pm 0,06$ \\
TCE & $2,29 \pm 0,35$ & $2,36 \pm 0,36$ & $2,43 \pm 0,07$ & $2,50 \pm 0,16$ \\
GV $(\%)$ & $1,19 \pm 0,12$ & $1,14 \pm 0,20$ & $1,29 \pm 0,18$ & $1,18 \pm 0,15$ \\
IHS $(\%)$ & $1,01 \pm 0,20$ & $1,01 \pm 0,12$ & $1,17 \pm 0,26$ & $0,96 \pm 0,14$ \\
& & & & \\
\hline
\end{tabular}

No hubo diferencia estadística $(p>0,05) . P I=$ peso inicial; $P F=$ peso final; $L T F=$ longitud total final; $S O B=$ supervivencia; $\mathrm{GP}=$ ganancia de peso; $\mathrm{CA}=$ conversión alimenticia; $\mathrm{TCE}=$ tasa de crecimiento específico; $\mathrm{GV}=$ grasa visceral; IHS= índice hepatosomático. 


\section{ACIDEZ DEL ACEITE DE PEZ EN LA ALIMENTACIÓN DE PACÚ}

industria, o por el poco tiempo de almacenamiento de los productos.

Boran et al. (2006) observaron que los aceites de pescado que generalmente presentan una alta concentración de ácidos grasos poliinsaturados son más susceptibles a procesos oxidativos, siempre dependiendo de las condiciones de almacenamiento. Es muy probable que los aceites empleados en este estudio y que mostraron un elevado índice de acidez permanecieron almacenados durante más tiempo, pero sin que los niveles de deterioro u oxidación llegaran a afectar el desempeño de los peces.

De acuerdo con Justi et al. (2003) la calidad de la dieta, así como los niveles de proteína y lípidos tiene una gran influencia en la composición corporal de los peces. Sin embargo, de acuerdo a los resultados en cuanto a composición de la carcasa que se presentan en la tabla III, no muestran variaciones en los niveles de grasa. Los diferentes grados de acidez del aceite utilizado no tuvieron ningún efecto sobre los niveles de materia seca, humedad, proteína, lípidos y cenizas (ANOVA, $\mathrm{p}>0,05$ ).

El porcentaje de agua de la carcasa del pacú fue de aproximadamente $73 \%$ para todos los tratamientos, mayor que lo presentado por Signor et al. (2010) para la misma especie, Reidel et al. (2010) para el jundia, e inferior a lo relatado por Signor et al. (2007) para el piavuçu. Así, las diferentes calidades de los aceites no influyeron en la deposición de los nutrientes en la carcasa.

No se observaron diferencias significativas en cuanto a los niveles de proteina, llegando a valores próximos al $14 \%$. Losekann et al. (2008) estudiando diferentes fuentes de aceites en la alimentación de jundia, tampoco encontraron diferencias, pero los niveles fueron más altos, lo que puede estar relacionado con la diferencia entre las especies y sus hábitos alimentícios. Bittencourt et al. (2010) aunque encontraron niveles más altos de proteína en la carcasa, no observaron diferencias significativas entre las diferentes densidades de estocaje de los animales.

Bombardelli et al. (2007) evaluaron la composición química del pacú criados en tanques de malla con tres dietas distintas y observaron que los niveles de lípidos de los animales varían en función del alimento suministrado. Los niveles de lípidos presentados por Bombardelli et al. (2007) son más bajos en el filete, pero mayores en el músculo dorsal y las espinas, cuando la alimentación se llevó a cabo usando dietas conteniendo desechos vegetales o desechos de pescado, mientras que en todos los casos los niveles de proteína son más altos a los obtenidos en el presente trabajo en todas las porciones analizadas. Signor et al.

Tabla III. Composición química de los juveniles de pacú alimentados con raciones que contienen aceites de diferentes índices de acidez. (Chemical composition of Piaractus mesopotamicus juveniles fed diets containing different levels of acidity).

\begin{tabular}{lcccc}
\hline & \multicolumn{4}{c}{ Índice de acidez de los aceites } \\
& 0,24 & 1,48 & 6,40 & 9,85 \\
\hline MS & $26,97 \pm 0,36$ & $26,97 \pm 0,52$ & $26,22 \pm 2,10$ & $26,65 \pm 0,51$ \\
UM & $73,03 \pm 0,36$ & $73,03 \pm 0,52$ & $73,78 \pm 2,10$ & $73,35 \pm 0,51$ \\
PB & $14,98 \pm 0,74$ & $14,51 \pm 0,24$ & $14,28 \pm 1,18$ & $14,39 \pm 0,41$ \\
LP & $8,60 \pm 0,40$ & $9,30 \pm 0,74$ & $8,75 \pm 1,22$ & $9,05 \pm 0,64$ \\
CZ & $3,83 \pm 0,25$ & $3,61 \pm 0,23$ & $3,48 \pm 0,33$ & $3,50 \pm 0,36$ \\
\hline
\end{tabular}

$\mathrm{MS}=$ materia seca $; \mathrm{UM}=$ humedad $\mathrm{PB}=$ proteína bruta; $\mathrm{LP}=$ lipídeos $; \mathrm{CZ}=$ cenizas. 


\section{LUI, BITTENCOURT, NEU, DALLAGNOL, BOSCOLO Y FEIDEN}

(2010) también observaron niveles de lípidos menores, siendo los niveles de proteína similares a los observados en este experimento.

Variaciones en la composición proximal de los peces pueden darse en función del estado nutricional del pez, así como de la edad y la densidad de estocaje. Sin embargo, niveles más elevados de lípidos dan lugar a un sabor más agradable en la carne, aunque reducen la vida útil del producto.

Con respecto a los parámetros hematológicos, que se presentan en la tabla IV, tanto el colesterol como los triglicéridos no mostraron diferencias significativas $(p>$ $0,05)$ entre los tratamientos.

El colesterol es producido en el hígado a partir de las grasas saturadas de la dieta, sin embargo, en este estudio se utilizaron, además de aceite de soja, 3 aceites de tilapia, ricos en ácidos grasos esenciales y poliinsaturados, lo que posiblemente no afectó la producción de colesterol o triglicéridos.

Los parámetros bioquímicos de la sangre de los peces son relativamente desconocidos, variando su composición en función de la especie, la alimentación y la forma como el animal está confinado. Sin embargo, los valores de colesterol y triglicéridos observados para el pacú son inferiores a los observados por Talas y Gulhan (2009) para la trucha arco-iris alimentados con propolis. Borges et al. (2004) evaluando los índices bioquímicos del jundia encontraron cantidades de colesterol que varían entre 110 y $240 \mathrm{mg} . \mathrm{dl}^{-1}$ y los triglicéridos entre 138 y 546 mg.dl ${ }^{-1}$, también superiores al encontrado en el estudio actual. Para las tilapias híbridas Hrubec et al. (2000) encontraron niveles de

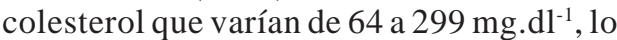
mismo fue observado por Melo et al. (2006) que encontraron niveles de triglicéridos en los jundias alimentados con diferentes fuentes proteicas elevados, lo que caracteriza las adaptaciones bioquímicas de la glucosa y gluconeogénesis para mantener los procesos de obtención de energía.

Para la misma especie, Hilbig et al. (2010) alimentándolos con diferentes tasas de ali-
Tabla IV. Niveles sanguíneos de colesterol y triglicéridos en los peces alimentados con niveles distintos de acidez del aceite. (Blood levels of cholesterol and triglycerides in fish fed diets containing oil with different levels of acidity).

\begin{tabular}{lccccc}
\hline \multicolumn{5}{c}{ Índice de acidez de los aceites } \\
\hline Variables & 0,24 & 1,48 & 6,40 & 9,85 & CV\% \\
Colesterol & 53,98 & 56,51 & 54,7 & 53 & $13,78^{\text {ns }}$ \\
Triglicerídos & 36,67 & 46,91 & 39,25 & 45,62 & $31,04^{\text {ns }}$ \\
\hline ns: no significativo. & & & \\
\hline
\end{tabular}

mentación analizaron los parámetros hematológicos y bioquímicos, y encontraron resultados superiores al del estudio actual, con niveles de colesterol y triglicéridos de 170 y $300 \mathrm{mg} \mathrm{dl}^{-1}$, respectivamente, sin embargo, los animales eran adultos, presentando una mayor acumulación de grasa perivisceral y de reservas nutricionales, en comparación con los peces más pequeños que están creciendo y utilizan toda la energía del alimento para sus funciones metabólicas normales.

Los exámenes hematológicos son necesarios para caracterizar fisiológicamente una especie, a través de su cuadro de comportamiento normal, en su medio ambiente natural, y luego, extrapolar estos resultados para los trabajos de campo (Ranzani-Paiva et al., 1999). Los peces de la presente investigación presentaron desempeño satisfactorio para la edad y en el período en que fueron sometidos a la prueba con diferentes índices de acidez de aceites, sin comprometer la salud de los peces, verificado por la ausencia de mortalidad y por los bajos índices de colesterol y triglicéridos.

\section{CONCLUSIÓN}

El uso de aceite de pescado con distinto nivel de acidez en la alimentación de juveniles de pacú no provoca resultados negativos ni en el desempeño productivo ni en el estado de salud de los animales. 


\section{ACIDEZ DEL ACEITE DE PEZ EN LA ALIMENTACIÓN DE PACÚ}

\section{BIBLIOGRAFÍA}

AOAC. 2005. Official methods of analysis of the AOAC. 18 ed. Association Of Official Analytical Chemists. Gaithersburg, M.D. USA.

Araújo, J.B., Severino, L.S., Lucena, A.M.A., Oliveira Freire, M.A., Guimarães, M.M.B. e Beltrão, N.E.M. 2006. Índice de acidez do óleo de quatro cultivares de mamona extraído por mini prensa laboratorial. Em: Anais do 20 Congresso Brasileiro de Mamona. Aracajú, SE.

Bittencourt, F., Feiden, A., Signor, A.A., Boscolo, W.R., Lorenz, E.K. e Maluf, M.L.F. 2010. Densidade de estocagem e parâmetros eritrocitários de pacus criados em tanquesrede. Rev Bras Zootecn, 39: 2323-2329.

Bombardelli, R.A., Bencke, B.C. e Sanches, E.A. 2007. Processamento da carne do pacu (Piaractus mesopotamicus) cultivado em tanques-rede no reservatório de Itaipu. Acta Sci Anim Sci, 29: 457-463.

Boran, G., Karaçam, H. and Boran, M. 2006. Changes in the quality of fish oils due to storage temperature and time. Food Chem, 98: 693698.

Borges, A., Scotti, L.V., Siqueira, D.R., Jurinitz, D.F. and Wassermann, G.F. 2004. Hematologic and serum biochemical values for jundiá ( Rham dia quelen). Fish Physiol Biochem, 30: 21-25.

Boscolo, W.R., Hayashi, C., Meurer, F., Feiden, A. e Wolff, L. 2004. Desempenho e características de carcaça de tilápias do Nilo (Oreochromis niloticus L.) alimentadas com rações contendo diferentes níveis de gordura. Rev Bras Zootecn, 26: 224-447.

Boyd, C. 1990. Water quality in ponds for aquaculture. Birmingham Publishing Co. London.

Cheng, Z.J., Hardy, R.W. and Usry, J.L. 2003. Effect of lysine supplementation in plant proteinbased diets on the performance of rainbow trout (Oncorhynchus mykiss) and apparent digestibility coefficients of nutrients. Aquaculture, 215: 255-265.

Graeff, A. e Tomazelli, A. 2007. Fontes e níveis de óleos na alimentação de carpa comum (Cyprinus carpio L.) na fase de crescimento. Ciênc Agrotec, 31: 1545-1551.

Hilbig, C., Zaminham, M., Dieterich, F., Maluf, M.L.F., Boscolo, W.R. e Feiden, A. 2010. Hematologia do pacu criado em tanques-rede submetido a diferentes taxas de alimentação. Em: II Simpósio Nacional de Engenharia de Pesca. Setembro, 2010. Toledo PR.

Hrubec, T.C., Cardinale, J.L. and Smith, S.A. 2000. Hematology and plasma chemistry reference intervals for cultured tilapia (Oreochromis hybrid). Vet Clin Phath, 29: 7-12.

Justi, K.C., Hayashi, C., Visentainer, J.C., Souza, N.E. and Matsushita, M. 2003. The influence of feed supply time on the fatty acid profile of Nile tilapia (Oreochromis niloticus) fed on a diet enriched with n-3 fatty acids. Food Chem, 80: 489-493.

Losekann, M.E., Radünz Neto, J., Emanuelli, T., Pedron, F.A., Lazzari, R., Bergamin, G.T., Corrêia, V. e Simôes, R.S. 2008. Alimentação do jundiá contendo óleos de arroz, canola ou soja. Ciênc Rural, 38: 225-230.

Melo, J.F.B., Tavares-Dias, M., Lundestedt, L.M. e Moraes, G. 2006. Efeito do conteúdo de proteína na dieta sobre os parâmetros hematológicos e metabólitos do bagre sul americano Rhamdia quelen. Rev Cienc Agroambientales, 1: 43-51.

Meurer, F., Hayashi, C., Boscolo, W.R. e Soares, C.M. 2002. Lipídeos na alimentação de alevinos revertidos de tilápia do Nilo (Oreochromis niloticus L.). Rev Bras Zootecn, 31: 566-573.

MPA. 2010. Ministério da Pesca e Aquicultura. Produção pesqueira e aquícola: Estatística 2008 e 2009. Brasilia, DF. 30 pp.

Ranzani-Paiva, M.J.T., Salles, F.A., Eiras, J.C., Eiras, A.C., Ishikawa, M.C. e Alexandrino, A.C. 1998/1999. Análises hematológicas de curimbatá (Prochilodus scrofa), pacu (Piaractus mesopotamicus) e tambaqui (Colossoma macropomum) das estações de piscicultura do Instituto de Pesca. Estado de São Paulo. Bol Instit Pesca, 25: 77-83.

Reidel, A., Romagoza, E., Feiden, A., Boscolo, W.R., Coldebella, A. e Signor, A.A. 2010. Rendimento corporal e composição química de jundiás alimentados com diferentes níveis de proteína e energia na dieta, criados em tanquesrede. Rev Bras Zootecn, 39: 233-240.

Signor, A.A., Boscolo, W.R., Feiden, A., Reidel, A., Signor, A. e Grosso, I.R. 2007. Farinha de vísceras de aves na alimentação de alevinos de piavuçu (Leporinus macrocephalus). Ciênc

Archivos de zootecnia vol. 61, núm. 236, p. 523. 


\section{LUI, BITTENCOURT, NEU, DALLAGNOL, BOSCOLO Y FEIDEN}

Rural, 37: 828-834.

Signor, A.A., Boscolo, W.R., Feiden, A., Bittencourt, B., Coldebella, A. e Reidel, A. 2010. Proteína e energia na alimentação de pacus criados em tanques-rede. Rev Bras Zootecn, 39: 23362341.

Sipaúba-Tavares, L.H. 1995. Limnologia aplicada à aquicultura. Funep. Jaboticabal. 72 pp.

Talas, Z.S. and Gulhan, M.F. 2009. Effects of various propolis concentrations on biochemical and hematological parameters of rainbow trout (Oncorhynchus mykiss). Ecotox Environ Safety, 72: 1994-1998.

Urbinati, E.C. e Gonçalves, F.D. 2005. Pacu (Piaractus mesopotamicus). Em: Baldisseroto, B., Gomes, L.C. Espécies nativas para piscicultura no Brasil. Editora da UFSM. Santa Maria, RS. 470 pp. 